\title{
New Mechanism for Electronic Energy Relaxation in Nanocrystals
}

\author{
Ho-Soon Yang, Michael R. Geller, and W. M. Dennis \\ Department of Physics and Astronomy, University of Georgia, Athens, Georgia 30602-2451
}

(May 28, 2000)

\begin{abstract}
The low-frequency vibrational spectrum of an isolated nanometer-scale solid differs dramatically from that of a bulk crystal, causing the decay of a localized electronic state by phonon emission to be inhibited. We show, however, that an electron can also interact with the rigid translational motion of a nanocrystal. The form of the coupling is dictated by the equivalence principle and is independent of the ordinary electron-phonon interaction. We calculate the rate of nonradiative energy relaxation provided by this mechanism and establish its experimental observability.
\end{abstract}

PACS: $63.22 .+\mathrm{m}, 71.55 .-\mathrm{i}, 78.66 . \mathrm{Vs}$

The dominant mechanism for the low-energy decay of a localized electronic impurity state in a macroscopic semiconductor or insulator is usually phonon emission [1.2n. In an isolated nanometer-scale crystal, however, the reduced dimensionality causes a suppression of the vibrational density-of-states (DOS) at low energies [3]. In particular, in a spherical nanoparticle of diameter $d$ there will be an acoustic phonon energy gap $\Delta \omega$ of the order of $2 \pi v / d$, where $v$ is a characteristic sound velocity of the bulk crystal. The one-phonon energy relaxation rate of an electron in the excited state of a two-level system with energy spacing $\Delta \epsilon$ therefore vanishes (or is greatly suppressed) when $\Delta \epsilon$ is less than $\Delta \omega$ [4]. Indeed, a dramatic suppression of the phonon-induced exciton dephasing rate [5],6] and nonradiative relaxation rate [7] 9] has been observed in nanocrystalline systems.

Nanoparticles are usually coupled to an environment consisting of other nanoparticles, a glass or polymer support matrix, or a solid substrate, and this mechanical interaction can modify a nanoparticle's low-frequency vibrational spectrum [10]. Fig. 1 shows a transmission-electron-microscope image of a cluster of $15 \mathrm{~nm} \mathrm{Y}_{2} \mathrm{O}_{3}$ nanoparticles prepared by gas-phase condensation. Although little is known in detail about the effects of environmental interaction on electronic energy relaxation in nanoparticles [11], it is clear that for the case of a nanoparticle only weakly coupled to other nanoparticles or to a substrate, phonon emission will still be prohibited because the low-frequency modes introduced by interaction with the environment will involve mostly rigid center-of-mass (CM) motion of the nanoparticles, which produces no strain. For example, Fig. 2 shows the collective-mode DOS for a model (illustrated in the inset) of the nanoparticle cluster of Fig. 1. Although the cluster possesses modes at frequencies less than $\Delta \omega$, which is about $10 \mathrm{~cm}^{-1}$ for these nanoparticles, these modes cannot couple to an impurity state through ordinary electron-phonon interaction.

In this paper we propose a new nonradiative relaxation mechanism caused by the inertial coupling of an electron to the nanoparticle's translational CM motion. This interaction is present because an electron bound to an impurity center in an oscillating nanoparticle is in an accelerating reference frame, and, in accordance with Einstein's equivalence principle, it feels a fictitious time-dependent force. We shall show below that this relaxation mechanism is operative even at zero temperature, owing to the fact that quantum zero-point motion of the CM is sufficient to produce the fictitious force.

In what follows we shall analyze the simplest nanoparticle model displaying the new effect; more sophisticated models, as well as the influence of inertial coupling on other dynamical processes such as dephasing, are subjects of on-going investigations and will be discussed in future publications. The present model consists of a single nanoparticle of mass $M$ connected to a bulk substrate by a few atomic bonds. The effect of the substrate is to subject the nanoparticle to a one-dimensional harmonic oscillator potential $V=\frac{1}{2} M \Omega^{2} X^{2}$ with frequency $\Omega$, the $X$ direction being perpendicular to the plane of the substrate. However, because the $\mathrm{CM}$ motion is that of a macroscopic harmonic oscillator interacting with many other degrees of freedom, such as the phonons of the bulk substrate, it is necessary on physical grounds to include energy dissipation (friction) of that oscillator. Possible rotational motion of the nanoparticle is not important here and will be ignored [12].

Denoting the CM of the nanoparticle by $\mathbf{R}$, a Hamiltonian in the CM frame can be obtained by rewriting the time-dependent Schrödinger equation in terms of new coordinates $\mathbf{r}^{\prime}=\mathbf{r}-\mathbf{R}$ and $t^{\prime}=t$. After a series of gauge transformations we obtain

$$
H_{\mathrm{CM}}=\sum_{\alpha} \epsilon_{\alpha} c_{\alpha}^{\dagger} c_{\alpha}+\sum_{n} \omega_{n} a_{n}^{\dagger} a_{n}+\Omega b^{\dagger} b+\sum_{n \alpha \alpha^{\prime}} g_{n \alpha \alpha^{\prime}} c_{\alpha}^{\dagger} c_{\alpha^{\prime}}\left(a_{n}+a_{n}^{\dagger}\right)-g \sum_{\alpha \alpha^{\prime}} x_{\alpha \alpha^{\prime}} c_{\alpha}^{\dagger} c_{\alpha^{\prime}}\left(b+b^{\dagger}\right)+\Delta H .
$$

The first term in Eqn. (1) is the Hamiltonian for a noninteracting two-level system; the other electronic levels can be neglected with no loss of generality. Here $\epsilon_{\alpha}$ (with $\alpha=1,2$ ) are the energy levels of the localized impurity state, $c_{\alpha}^{\dagger}$ and $c_{\alpha}$ are electron creation and annihilation operators [13]. The second term in Eqn. (11) describes the 
nanoparticle's internal vibrational dynamics. The $\omega_{n}$ are the frequencies of the internal modes, and the $a_{n}^{\dagger}$ and $a_{n}$ are the corresponding phonon creation and annihilation operators. For the case of a perfectly spherical nanoparticle the vibrational eigenmodes and eigenvalues can be obtained analytically within continuum elasticity theory [14, 15]; the frequency of the lowest internal mode (a 5 -fold degenerate torsional mode) is approximately $2 \pi v_{\mathrm{t}} / d$, where $v_{\mathrm{t}}$ is the bulk transverse sound velocity and $d$ is the diameter. These internal vibrational modes have been observed by low-frequency Raman scattering [16 20] and by femptosecond pump-probe spectroscopy [21].

The third term in Eqn. (11) describes the harmonic dynamics of the CM. As discussed above, the nanoparticle is assumed to be constrained to move in the $x$ direction only. Hence, the CM translational motion is described by a single bosonic degree-of-freedom,

$$
b \equiv \sqrt{\frac{M \Omega}{2}}\left(X+\frac{i}{M \Omega} P\right)
$$

where $X$ and $P$ are the $x$-components of the CM position and momentum.

The fourth term in $H_{\mathrm{CM}}$ is the ordinary leading-order interaction between the two-level system and the internal vibrational modes. Here $g_{n \alpha \alpha^{\prime}}$ is the electron-phonon coupling constant; it depends on the detailed microscopic structure of the nanoparticle, the nature and position of the impurity, and the spatial dependence of the internal vibrational modes. In the regime of interest here, where phonon emission is inhibited, this electron-phonon interaction term can be ignored, and the remaining Hamiltonian is that of a two-level atom in a cavity with a single damped mode 22].

The fifth term in Eqn. (11), which describes the inertial coupling between the two-level system and the CM motion, is the focus of the present work. Here $x_{\alpha \alpha^{\prime}} \equiv\left\langle\phi_{\alpha}|x| \phi_{\alpha^{\prime}}\right\rangle$ are dipole-moment matrix elements, which, of course, depend on the form of the impurity states $\phi_{\alpha}(\mathbf{r})$, and

$$
g \equiv \sqrt{\frac{m^{2} \Omega^{3}}{2 M}}
$$

is a coupling constant that depends only on the (bare) electron mass $m$ and on macroscopic properties of the nanoparticle. The presence of this fifth term can be understood as follows: The Hamiltonian for the noninteracting two-level system, the first term in $H_{\mathrm{CM}}$, is written in a coordinate system moving with the oscillating nanoparticle, which is a noninertial reference frame. According to the equivalence principle [23], the electron therefore sees an additional uniform force

$$
\mathbf{F}_{\text {eff }}=-m \ddot{\mathbf{R}}
$$

where, as before, $\mathbf{R}$ is the nanoparticle CM. For the case of harmonic motion, Eqn. (4) can be written as $\mathbf{F}_{\text {eff }}=m \Omega^{2} \mathbf{R}$. Thus, the potential energy of an electron at position $\mathbf{r}$ in the $\mathrm{CM}$ frame is

$$
U=-m \Omega^{2} \mathbf{R} \cdot \mathbf{r},
$$

which, in one dimension, is equivalent to the fifth term in Eqn. (1). Although spin indices have been suppressed in Eqn. (1), it should be understood that the inertial coupling between the electron and the CM motion conserves spin.

The final term in Eqn. (11), denoted by $\Delta H$, describes an interaction between the nanoparticle's translational motion and a bath of other harmonic oscillators, such as the phonons of the bulk substrate or the CM degrees-of-freedom of other nanoparticles [24]. The effect of $\Delta H$ is to dissipate energy from the oscillating nanoparticle. In the absence of this damping, the inertial coupling causes energy to be continuously exchanged between the two-level system and the CM oscillator in a Rabi-like fashion; this interesting dissipation-free limit, although not relevant for the nanoparticle systems considered here, will be discussed in detail elsewhere.

To study the effect of the fifth term in Eqn. (11) on the electronic energy relaxation rate we calculate the electron self-energy perturbatively 25. We assume that $\Delta \epsilon$ is sufficiently smaller than $\Delta \omega$ so that ordinary phonon emission is prohibited; this allows us to set $g_{n \alpha \alpha^{\prime}}=0$. The leading-order self-energy is

$$
\Sigma(\alpha, i \omega)=-\frac{g^{2}}{\beta} \sum_{\omega_{\mathrm{B}}} \sum_{\alpha^{\prime}}\left|x_{\alpha \alpha^{\prime}}\right|^{2} G_{0}\left(\alpha^{\prime}, i \omega-i \omega_{\mathrm{B}}\right) D\left(i \omega_{\mathrm{B}}\right),
$$

where $\beta$ is the inverse temperature, $\omega_{\mathrm{B}}$ is a bosonic Matsubara frequency, $G_{0}(\alpha, i \omega)=\left(i \omega-\epsilon_{\alpha}\right)^{-1}$ is the noninteracting electron Green's function, and $D\left(i \omega_{\mathrm{B}}\right)$ is the Fourier transform of a phonon propagator

$$
D(\tau) \equiv-\langle T[b(\tau)+\bar{b}(\tau)][b(0)+\bar{b}(0)]\rangle
$$


that has been renormalized to include the effects of $\Delta H$. The precise form of $D\left(i \omega_{\mathrm{B}}\right)$ depends, of course, on $\Delta H$, and in the absence of a reliable microscopic model for $\Delta H$ we shall use a (retarded) propagator of the form

$$
D^{R}(\omega)=\frac{1}{\omega-\Omega+i \gamma}-\frac{1}{\omega+\Omega+i \gamma}
$$

which has a Lorentzian spectral function of width $\gamma$.

The relaxation rate $\tau^{-1} \equiv-\left.2 \operatorname{Im} \Sigma^{R}\left(\alpha, \epsilon_{\alpha}\right)\right|_{\alpha=2}$ of the excited state at zero temperature is found to be

$$
\tau^{-1}=2 \pi g^{2}\left|x_{12}\right|^{2} f(\Delta \epsilon-\Omega),
$$

where $f(\omega) \equiv \gamma / \pi\left(\omega^{2}+\gamma^{2}\right)$ is a Lorentzian function of width $\gamma$. The fact that relaxation occurs even at zero temperature, when the nanoparticle $\mathrm{CM}$ is in its ground state, shows that zero-point CM motion is sufficient to produce a fictitious force.

When the energy separation $\Delta \epsilon$ between the two levels is resonant with the frequency $\Omega$ of the translational mode, the electronic relaxation rate becomes

$$
\tau_{\text {res }}^{-1}=4 g^{2}\left|x_{12}\right|^{2} \tau_{\mathrm{cm}},
$$

where $\tau_{\mathrm{cm}} \equiv 1 / 2 \gamma$ is the lifetime of the translational mode. This can be written (reinstating factors of $\hbar$ ) as

$$
\tau_{\text {res }}^{-1} \approx 1.6 \times 10^{-21} \frac{Q \Omega_{0}^{2} X^{2}}{M} s^{-1}
$$

where $Q \equiv \tau_{\mathrm{cm}} \Omega$ is the quality factor of the $\mathrm{CM}$ oscillator, $\Omega_{0}$ is the $\mathrm{CM}$ oscillation frequency in measured in wavenumbers, $X$ is the dipole moment measured in Bohr radii, and $M$ is the nanoparticle mass measured in grams.

We believe that it should be possible to observe this novel relaxation mechanism by means of an infrared quantum counter experiment [26] in $\mathrm{LaF}_{3}: \mathrm{Ho}^{3+}$ nanoparticles. The $\mathrm{Ho}^{3+}{ }^{5} \mathrm{I}_{8}$ (II) level is $4.5 \mathrm{~cm}^{-1}$ above the ground state and can be excited using a pulsed far infrared source. The population of the ${ }^{5} \mathrm{I}_{8}$ (II) state can be probed by a pulsed visible laser tuned to the ${ }^{5} \mathrm{I}_{8}$ (II) $\rightarrow{ }^{5} \mathrm{~S}_{2}$ (I) transition; a measurement of the intensity of the ${ }^{5} \mathrm{~S}_{2}$ (I) emission as a function of the delay between the far infrared and optical pulses would enable the relaxation of the ${ }^{5} \mathrm{I}_{8}(\mathrm{II})$ to be observed. The resonant relaxation rate for $\mathrm{LaF}_{3}$ nanoparticle with $10 \mathrm{~nm}$ diameter is approximately

$$
\tau_{\text {res }}^{-1} \approx 1.0 \times 10^{-2} \mathrm{~s}^{-1} \quad \text { for } Q=10^{2}
$$

and

$$
\tau_{\text {res }}^{-1} \approx 1.0 \times 10^{2} \mathrm{~s}^{-1} \quad \text { for } Q=10^{6} .
$$

The second estimate applies to a very weakly damped nanoparticle. It is simple to show that, for this nanoparticle, the maximum $Q$ factor allowed by the perturbative analysis leading to Eqn. (9) is about $10^{8}$ (see footnote [25]). Although small, these rates are still much larger than the radiative rate between these closely spaced levels.

In conclusion, our analysis shows that fictitious forces produce a coupling between an impurity state in a doped nanoparticle and the rigid-body CM motion. If the CM oscillation frequency is near (on the scale of $\gamma$ ) to the level separation $\Delta \epsilon$, this effect provides a mechanism for energy relaxation even when conventional phonon emission is prohibited. We find that on resonance the relaxation rate is proportional to the translational mode lifetime $\tau_{\mathrm{cm}}$ : This means that relaxation is faster when the translational mode is weakly coupled to its environment, whereas coupling to an appreciably damped CM mode is less effective. Although we do not believe that the mechanism proposed here is responsible for the energy relaxation observed in Refs. [8] and [9], our estimates clearly show that this somewhat exotic phenomena is experimentally accessible.

This work was supported by a Research Innovation Award from the Research Corporation and by National Science Foundation Grant No. DMR-9871864. It is a pleasure to thank Uwe Happek, David Huber, Coates Johnson, Vadim Markel, Richard Meltzer, and William Yen for useful discussions.

[1] B. Henderson and G. F. Imbusch, Optical Spectroscopy of Inorganic Solids (Clarendon Press, Oxford, 1989). 
[2] H. Kuzmany, Solid-State Spectroscopy: An Introduction (Springer-Verlag, Berlin, 1998).

[3] S. V. Gaponenko, Optical Properties of Semiconductor Nanocrystals (Cambridge University Press, Cambridge, 1998).

[4] This statement is correct, of course, only when there is some intrinsic or extrinsic phonon broadening mechanism that makes Fermi's golden rule applicable.

[5] K. Misawa, H. Yao, T. Hayashi, and T. Kobayashi, Chem. Phys. Lett. 183, 113 (1991).

[6] K. Misawa, H. Yao, T. Hayashi, and T. Kobayashi, J. Chem. Phys. 94, 4131 (1991).

[7] U. Woggon, S. V. Gaponenko, A. Uhrig, W. Langbein, and C. Klingshirn, Adv. Mat. Opt. Electron. 3, 141 (1994).

[8] H. S. Yang, S. P. Feofilov, D. K. Williams, J. C. Milora, B. M. Tissue, R. S. Meltzer, and W. M. Dennis, Physica B 263, 476 (1999).

[9] H. S. Yang, K. S. Hong, S. P. Feofilov, B. M. Tissue, R. S. Meltzer, and W. M. Dennis, J. Lumin. 83, 139 (1999).

[10] For a survey of novel nanocrystal geometries, see A. P. Alivisatos, Science 271, 933 (1996), and references within.

[11] An exception is the case of nanocrystals embedded in a glass matrix, which has been studied by M. Montagana and R. Dusi, Phys. Rev. B 52, 10080 (1995), and by N. N. Ovsyuk and V. N. Novikov, Phys. Rev. B 53, 3113 (1996).

[12] Rotation would lead to an fictitious time-dependent magnetic field acting on the electron, which is less effective at inducing an electronic transition.

[13] The electrons are taken to be spinless for simplicity.

[14] H. Lamb, Proc. Math. Soc. London 13, 187 (1882).

[15] See also T. Takagahara, J. Lumin. 70, 129 (1996), and references within.

[16] E. Duval, A. Boukenter, and B. Champagnon, Phys. Rev. Lett. 56, 2052 (1986).

[17] B. Champagnon, B. Andrianasolo, and E. Duval, J. Chem. Phys. 94, 5237 (1991).

[18] A. Tanaka, S. Onari, and T. Arai, Phys. Rev. B 47, 1237 (1993).

[19] T. D. Krauss, F. W. Wise, and D. B. Tanner, Phys. Rev. Lett. 76, 1376 (1996).

[20] T. D. Krauss and F. W. Wise, Phys. Rev. Lett. 79, 5102 (1997).

[21] G. Cerullo, S. De Silvestri, and U. Banin, Phys. Rev. B 60, 1928 (1999).

[22] M. O. Scully and M. S. Zubairy, Quantum Optics (Cambridge University Press, Cambridge, 1997).

[23] See, for example, A. Einstein, The Meaning of Relativity, 5th ed. (Princeton University Press, Princeton, 1956).

[24] It is implied that $\Delta H$ includes the kinetic and potential energies of the bath degrees of freedom as well.

[25] A rotating-wave analysis shows that perturbation theory is valid here when the energy width $\gamma$ of the CM translational mode is larger than the interaction energy $g\left|x_{12}\right|$, as expected by dimensional considerations.

[26] N. Bloembergen, Phys. Rev. Lett. 2, 84 (1959).

FIG. 1. Cluster of $\mathrm{Y}_{2} \mathrm{O}_{3}$ nanoparticles. The mean particle diameter is approximately $15 \mathrm{~nm}$.

FIG. 2. Histogram of the number of vibrational modes per unit frequency of a model nanoparticle cluster, shown in the inset. The frequency is given in wavenumbers. The cluster contains about 1300 nanoparticles of identical mass $M$, and between each adjoining nanoparticle is a harmonic spring with a stiffness $k$ chosen such that $\sqrt{k / M}$ is equal to $1 \mathrm{~cm}^{-1}$. 